\title{
Quantitative analysis of axonal loss in band atrophy of the optic nerve using scanning laser polarimetry
}

\author{
M L R Monteiro, F A Medeiros, M R Ostroscki
}

Br J Ophthalmol 2003;87:32-37

Aims: To measure axonal loss in patients with band atrophy from optic chiasm compression using scanning laser polarimetry (GDx, Laser Diagnostic Technologies, Inc, San Diego, CA, USA) and to evaluate the ability of this instrument to identify this pattern of retinal nerve fibre layer (RNFL) loss.

Methods: 19 eyes from 17 consecutive patients with band atrophy of the optic nerve and permanent temporal hemianopia due to chiasmal compression, and 19 eyes from an age and sex matched control group of 17 healthy individuals were prospectively studied. All patients were submitted to an ophthalmic examination including Goldmann perimetry and evaluation of the RNFL using scanning laser polarimetry. Mean RNFL thickness around the optic disc were compared between the two groups. The diagnostic performance of the deviation from normal analysis provided by the GDx software was also assessed. Results: The peripapillary RNFL thickness (mean (SD)) of eyes with band atrophy was 47.9 (7.63) $\mu \mathrm{m}, 37.1$ (8.48) $\mu \mathrm{m}, 57.0(9.31) \mu \mathrm{m}$, and $37.2(8.86) \mu \mathrm{m}$ in the superior, temporal, inferior, and nasal regions, respectively. The total average was 43.7 (12.0) $\mathrm{\mu m}$. In the control group, the corresponding values were 71.1 (12.2) $\mu \mathrm{m}, 40.4$ (10.9) $\mu \mathrm{m}, 85.4(14.0) \mu \mathrm{m}$, and 49.8 (10.1) $\mu \mathrm{m}$. The total average measured 67.9 (11.2) $\mu \mathrm{m}$. The measurements from eyes with optic atrophy were significantly different from those in the control group in all regions but the temporal. The deviation from normal analysis provided by the GDx software failed to identify the majority of abnormalities in the temporal and nasal regions of patients with band atrophy.

Conclusions: Scanning laser polarimetry was able to identify axonal loss in the superior, inferior, and nasal regions, but failed to detect it in the temporal region of the optic disc, despite the fact that this area was clearly altered in eyes with band atrophy. This examination also showed poor sensitivity to detect axonal loss in the nasal region when GDx software analysis was used. The results of this study emphasise that RNFL evaluation using scanning laser polarimetry should be interpreted with caution in the study of eye diseases that lead to axonal loss predominantly in the nasal and temporal areas of the optic disc.

$\mathrm{T}$ he GDx nerve fibre analyser (Laser Diagnostic Technologies, Inc, San Diego, CA, USA) is a scanning laser polarimeter designed to assess the peripapillary nerve fibre layer thickness in vivo. Its ability to provide quantitative and reproducible measurements of the retinal nerve fibre layer (RNFL) has been demonstrated in experimental and clinical studies. ${ }^{1-7}$ Although most of the clinical studies using GDx have been performed on glaucoma patients, many other optic neuropathies have been studied including demyelinating optic neuritis, ${ }^{8}$ indirect optic nerve trauma, ${ }^{9}$ ischaemic optic neuropathy, ${ }^{10}$ and retrobulbar optic neuropathies. ${ }^{11}$ In many of these conditions the findings of nerve fibre loss on GDx is sometimes difficult to distinguish from false positive results. Although correlation with visual field defects is helpful in such cases, studies of conditions such as glaucoma can be difficult to interpret as the nerve fibre loss in this condition may not follow a specific pattern and, in most cases, is difficult to predict clinically.

Previous reports have shown good correlation between visual field defects and GDx measurements obtained in the superior and inferior peripapillary areas ${ }^{12}$; however, the ability of this instrument to assess RNFL thickness in the nasal and temporal regions has been questioned. ${ }^{13}{ }^{14}$ The pattern of nerve fibre layer loss in patients with optic chiasm compression may represent a model to evaluate the ability of the GDx to measure nerve fibre layer loss in the nasal and temporal regions of the optic disc. Patients with extensive mid-chiasmal lesions affecting crossed nerve fibres, and showing complete or almost complete bitemporal hemianopia with preserved nasal field, generally demonstrate very distinctive changes in the peripapillary area. In these cases, the crossed nerve fibres (which have their cell bodies in the nasal hemiretina) are lost with preservation of the uncrossed nerve fibres, which originate in the temporal hemiretina and penetrate the optic nerve through the superior and inferior arcuate fibre bundles. Therefore, the RNFL loss occurs predominantly in the nasal and temporal side of the optic disc. Such a pattern is identified on ophthalmoscopy as band atrophy of the RNFL and is very important in the diagnosis of chiasmal compression and in estimating the chances of improvement of visual field defects after decompression of the optic pathway.

Unsold and Hoyt ${ }^{15}$ described the histological features of the optic nerve in a patient with band atrophy of the optic nerve and complete temporal hemianopia. There was complete loss of the nasal and temporal nerve fibres with relative preservation of the superior and inferior arcuate fibre bundles. Although Mildelberg and Yidegiligne ${ }^{16}$ performed automated quantitative histological analysis of an optic nerve with band atrophy, to our knowledge no study had been performed using scanning laser polarimetry in such cases. We therefore performed a prospective study using the GDx in patients with well defined temporal hemianopia and band atrophy of the optic nerve secondary to chiasm compression, in order to evaluate the ability of this instrument to identify the typical pattern of nerve fibre loss seen in these patients.

\section{METHODS}

Between January 1999 and February 2002, 19 eyes from 17 patients with complete or nearly complete temporal hemianopia from chiasmal compression and 19 eyes from 17 normal age and sex matched controls were submitted to scanning laser polarimetry of the peripapillary RNFL. Thirteen patients had pituitary adenoma, three patients had craniopharyngioma, and one had a chiasmal astrocytoma. All patients had 
Table 1 Age, sex, study eye, tumour diagnosis, and visual field defect in the 17 patients (19 eyes) with temporal hemianopia from chiasmal compression

\begin{tabular}{lllllll}
\hline Patient & Sex & Age & Eye & VA & Diagnosis & Visual field \\
\hline 1 & $M$ & 57 & RE & $20 / 20$ & Adenoma & Complete hemianopia \\
2 & $M$ & 41 & LE & $20 / 20$ & Craniopharyngeoma & Complete hemianopia \\
3 & $M$ & 37 & LE & $20 / 20$ & Adenoma & Complete hemianopia \\
3 & $M$ & 33 & LE & $20 / 30$ & Craniopharyngeoma & IT reminiscent with V/4e \\
5 & $F$ & 46 & RE & $20 / 20$ & Adenoma & IT reminiscent with V/4e \\
6 & F & 40 & RE & $20 / 20$ & Craniopharyngeoma & Complete hemianopia \\
7 & $M$ & 43 & RE & $20 / 20$ & Adenoma & IT reminiscent with V/4e \\
8 & $M$ & 21 & RE & $20 / 20$ & Astrocytoma & Complete hemianopia \\
9 & $M$ & 51 & RE & $20 / 30$ & Adenoma & IT reminiscent with V/4e \\
& & & LE & $20 / 20$ & & IT reminiscent with V/4e \\
10 & $M$ & 47 & LE & $20 / 20$ & Adenoma & Complete hemianopia \\
11 & $F$ & 45 & RE & $20 / 20$ & Adenoma & IT reminiscent with V/4e and I/4e \\
12 & $F$ & 36 & LE & $20 / 20$ & Adenoma & IT reminiscent with V/4e \\
13 & $M$ & 69 & LE & $20 / 30$ & Adenoma & IT reminiscent with V/4e \\
14 & $M$ & 62 & RE & $20 / 20$ & Adenoma & IT reminiscent with V/4e and I/4e \\
15 & $F$ & 20 & RE & $20 / 20$ & Adenoma & Complete hemianopia \\
16 & $M$ & 45 & RE & $20 / 20$ & Adenoma & IT reminiscent with V/4e \\
17 & $M$ & 41 & LE & $20 / 20$ & Adenoma & Complete hemianopia \\
\hline \multirow{2}{*}{ VA $=$ visual acuity $;$} & IT = inferior temporal. & & IT reminiscent with V/4e and I/4e \\
\hline
\end{tabular}

stable visual field defects and stable visual acuity for at least 6 months and had already been submitted to previous treatment of the suprasellar lesion before study entry. The patients underwent a comprehensive ophthalmological examination including best corrected visual acuity, applanation tonometry, pupillary reactions, extraocular motility evaluation, slit lamp examination, funduscopy and visual field evaluation. Dilated fundus examination was performed only after completion of visual field studies and scanning laser polarimetry.

Visual field testing was performed using the Goldmann perimeter (Haag-Streit AG, Bern, Switzerland). The V-4-e, I-4-e, I-3-e, I-2-e, and I-1-e stimuli were used to draw the isoptres. Kinetic determinations were followed by static presentation of the stimuli particularly in the central 30 degree area in order to search for localised defects. Five patients were also submitted to standard automated perimetry (Humphrey 24-2 full threshold test).

The inclusion criteria were best corrected visual acuity of $20 / 30$ or better in the study eye; age between 20 and 70 years, spherical refraction within plus or minus $3 \mathrm{D}$; cylinder correction within plus or minus $3 \mathrm{D}$; intraocular pressure less than $22 \mathrm{~mm} \mathrm{Hg}$; and reliable Goldmann visual fields. Patients with previous history of intraocular pressure elevation or with clinical signs of glaucomatous optic neuropathy (neuroretinal rim thinning, notching, excavation, or optic disc haemorrhage) were excluded.

The GDx nerve fibre analyser is a confocal scanning laser ophthalmoscope coupled with an integrated polarisation modulator. Details of its operation have been reported previously. ${ }^{2}$ The technique is based on birefringence properties of the RNFL. The ganglion cell axons contain microtubules, cylindrical intracellular organelles with diameters much smaller than the wavelength of the illuminating light, which are form birefringent. The RNFL birefringence induces a change (retardation) in the state of polarisation of an illuminating laser beam that passes through it. The retardation has been shown to be correlated to the thickness of the RNFL.

Three to six retardation maps of the peripapillary retina of each eye were acquired and a mean map was created and used for analysis by averaging the three best images. Examinations on eyes with undilated pupils were subsequently performed by the same experienced technician. The images were evaluated by both subjective and software generated image quality assessment. Poor quality images were discarded. The disc margin was established by an experienced operator using an ellipse to outline the inner margin of the peripapillary scleral ring. A 10 pixel wide elliptical band was automatically positioned concentrically to the disc margin outline and at 1.75 disc diameters from the centre of the optic disc. The elliptical band was divided into superior and inferior segments of 120 degrees each, a nasal segment of 70 degrees, and a temporal segment of 50 degrees. The average number of pixels in each of the four segments and in the full ellipse (total average) was calculated automatically with GDx software (ver 1.0.16). Measurements of RNFL thickness in each quadrant and in the full ellipse for eyes with band atrophy and controls were compared using Student's $t$ test. p Values of less than 0.05 were considered significant.

Another set of measurements, the deviation from normal values, were calculated for each quadrant using the average of the 1500 thickest points in the inferior and superior quadrants and the average of the median 1500 points in the temporal and nasal quadrants. The deviation from normal value represents the difference between the RNFL thickness measurement of the patient and the mean of the normal population. Negative values indicate that the RNFL thickness is inferior to that of the normal population. Along with the deviation from normal value of each quadrant, a probability value $(>10 \%,<10 \%$, $<5 \%,<2 \%$, or $<1 \%$ ) is also shown. This probability value is derived from a comparison with the normative database of the machine. The GDx software uses a race and age specific normative database of more than 1100 eyes to classify the measures of RNFL. According to the manufacturer, healthy patients aged 18-80 years were enrolled using a standard protocol for inclusion in the normative database. Healthy eyes could not have a significant visual field defect, unreliable field, or pathological appearance of the optic disc. The deviation

Table 2 Mean values (SD) of retinal nerve fibre layer thickness $(\mu \mathrm{m})$ in each segment for the 19 control eyes and 19 eyes with band atrophy

\begin{tabular}{llll}
\hline & Band atrophy $(\mathrm{n}=19)$ & Controls $(\mathrm{n}=19)$ & $\mathrm{p}$ Value* \\
\hline Total & $47.4(7.63)$ & $67.9(11.2)$ & $<0.0001$ \\
Superior & $47.9(7.63)$ & $71.1(12.2)$ & $<0.0001$ \\
Temporal & $37.1(8.48)$ & $40.4(10.9)$ & 0.30 \\
Inferior & $57.0(9.31)$ & $85.4(14.0)$ & $<0.0001$ \\
Nasal & $37.2(8.86)$ & $49.8(10.1)$ & 0.0002 \\
\hline \multirow{2}{*}{ *Student's $t$ test. Significant values are in italics. }
\end{tabular}




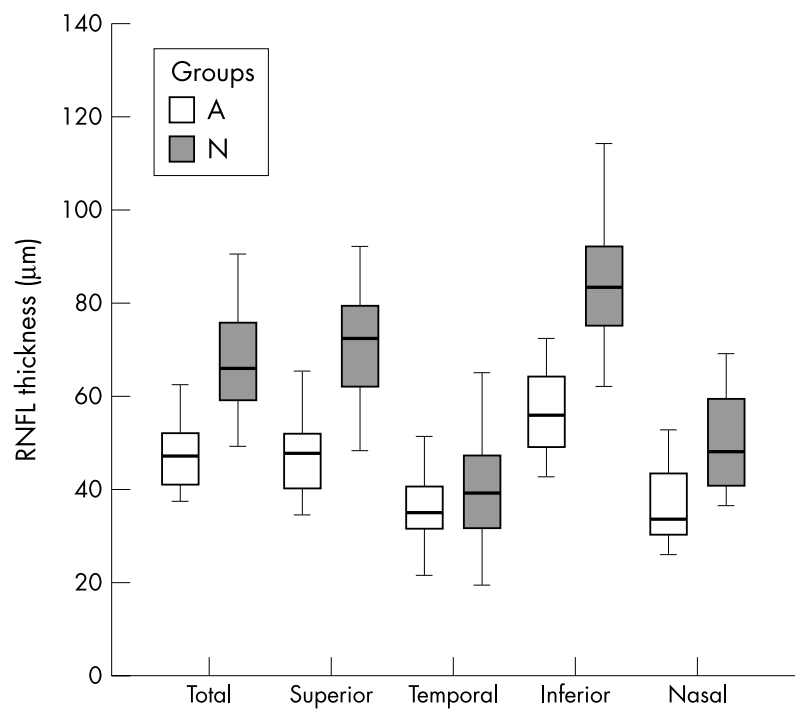

Figure 1 Box plot chart showing the distribution of retinal nerve fibre layer (RNFL) thickness measurements in each segment in both groups ( $\mathrm{A}=$ patients with band atrophy; $\mathrm{N}=$ normal controls). from normal values and associated probabilities are included in the GDx printout and provide an easy way for the clinician to rapidly assess RNFL loss in each quadrant. In this study, the GDx software classification of the deviation from normal values was evaluated for each patient in the two groups.

\section{RESULTS}

A total of 19 eyes with temporal hemianopia and 19 control eyes were studied. Data concerning age, sex, type of tumour, visual acuity and visual fields can be found in Table 1 . The study group and the control group both consisted of 12 male and five female patients. Mean age and standard deviation (SD) was 44.1 (11.9) years in the study group and 43.7 (12.0) years in the control group $(\mathrm{p}=0.92$; Student's $t$ test). Eight eyes had complete temporal hemianopia, up to V/4e target; and eight eyes had almost complete hemianopia with only a small inferior temporal reminiscent of field with V/4e target and a complete temporal defect up to I/4e target. Three eyes presented with a small temporal reminiscent of field with V/4e and I/4e target, and with complete defect up to the I/3e stimulus. The five eyes studied with Humphrey perimetry (eyes 4, 7, 10, 13, and 17) revealed complete temporal hemianopia. The nasal field was within normal limits in all studied eyes. Dilated funduscopic examination revealed band atrophy of the optic disc and RNFL in all 19 eyes with temporal hemianopia.
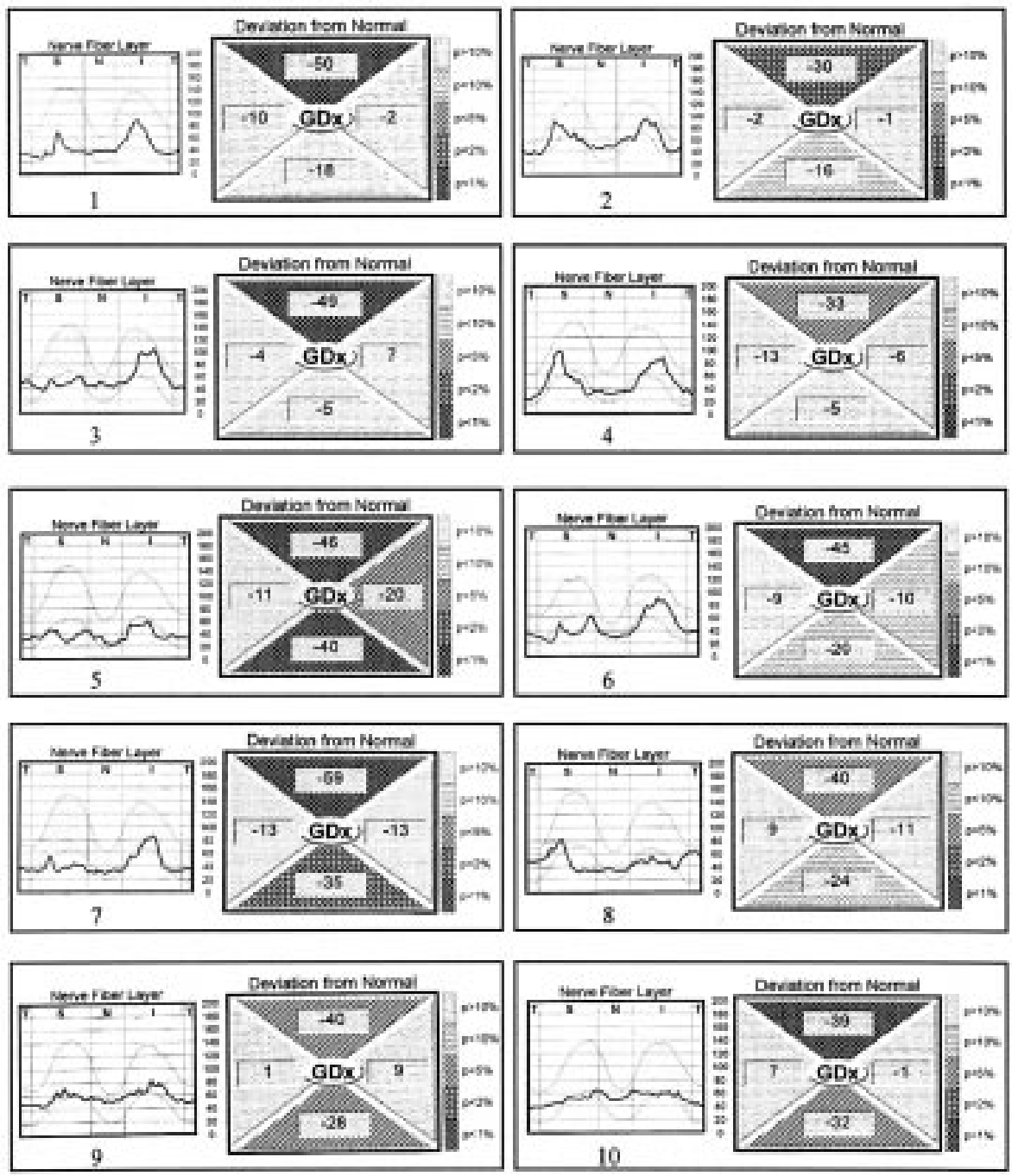

Figure 2 Retinal nerve fibre layer thickness profile and deviation from normal values in each segment, as presented in the GDx printout, for the 19 eyes with band atrophy. Part 1, eyes $1-10$. 

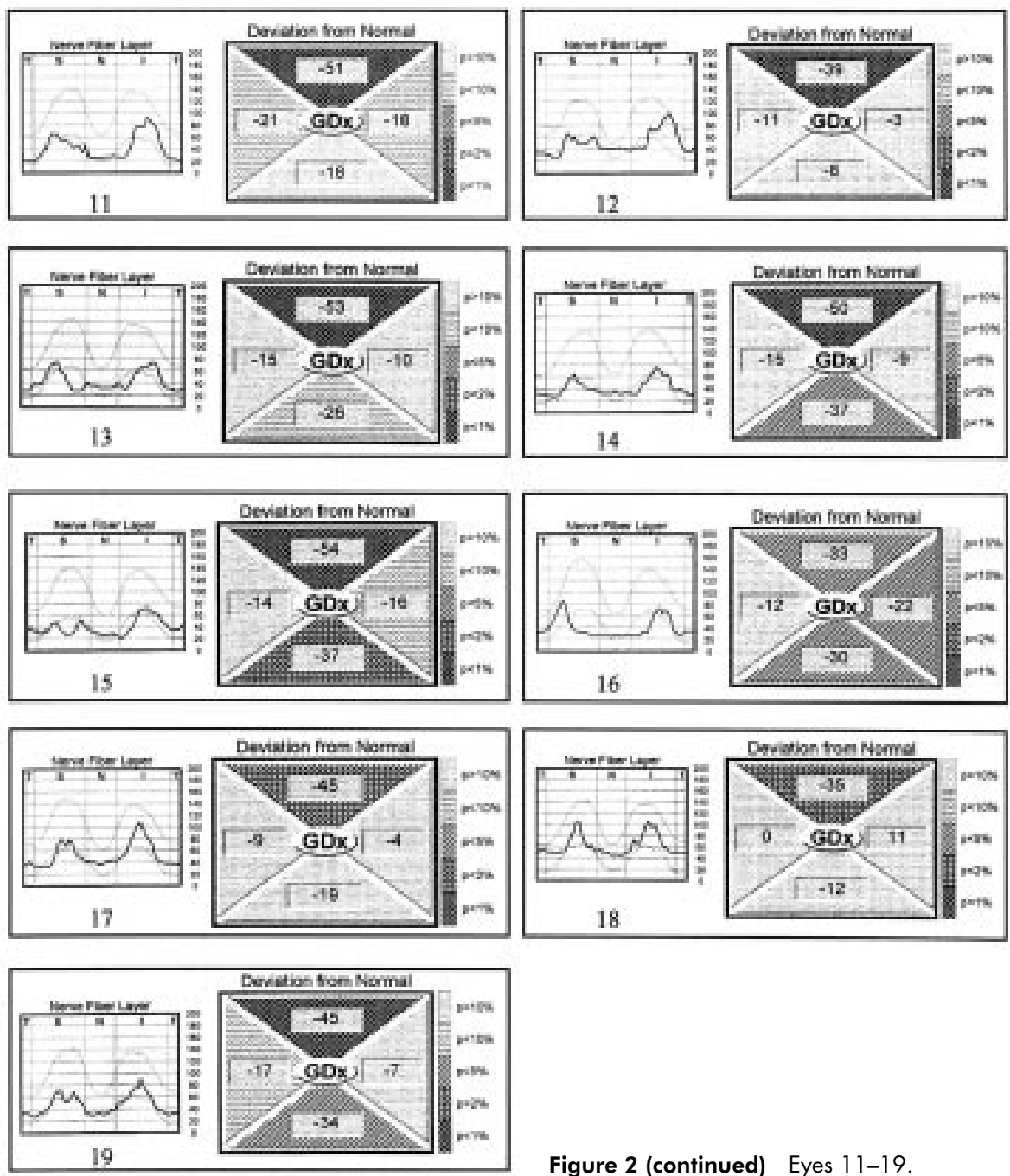

Figure 2 (continued) Eyes 11-19.

The mean peripapillary RNFL thickness of the eyes with band atrophy measured 47.9 (7.63) $\mu \mathrm{m}, 37.1$ (8.48) $\mu \mathrm{m}, 57.0$ (9.31) $\mu \mathrm{m}$, and $37.2(8.86) \mu \mathrm{m}$ in the superior, temporal, inferior, and nasal regions, respectively. The total average was 43.7 (12.0) $\mu \mathrm{m}$. In the control group, the mean RNFL thickness measured 71.1 (12.2) $\mu \mathrm{m}, 40.4$ (10.9) $\mu \mathrm{m}, 85.4$ (14.0) $\mu \mathrm{m}$, and 49.8 (10.1) $\mu \mathrm{m}$ in the superior, temporal, inferior, and nasal regions, respectively. The total average measured 67.9 (11.2) $\mu \mathrm{m}$. The total average, superior average, inferior average, and nasal average were found to be significantly lower in eyes with band atrophy compared with normal eyes. However, the values from the temporal region were not significantly different between the two groups (Table 2). Figure 1 shows the distribution of the RNFL thickness values in each segment for the two groups.

Figure 2 shows the RNFL thickness profile and deviation from normal values in each segment, as presented in the GDx printout, for the 19 eyes with band atrophy. Abnormality in the deviation from normal value (with probability of less than $5 \%$ of being normal) was observed in the superior region of the optic disc in all 19 eyes with band atrophy, in the inferior region of eight eyes, in the nasal region in only two eyes (eyes Nos 5 and 16), and in none in the temporal region (Fig 2). The same analysis in the control group showed abnormality in the superior region of the optic disc in one eye and in the nasal region of the disc in another eye.

\section{DISCUSSION}

Accurate evaluation of RNFL loss is extremely important in glaucoma as well as in many neuro-ophthalmological conditions. After the initial observation of Hoyt and Newman, ${ }^{17}$ in 1972, using direct ophthalmoscopy, numerous studies have emphasised its clinical importance. Although several non-invasive techniques ${ }^{18-20}$ have been used to assess the RNFL, it is still unclear whether these techniques effectively measure the RNFL thickness and how large the RNFL loss must be before it is detectable.

The GDx nerve fibre analyser is a promising instrument for the evaluation of the retinal nerve fibre layer. It provides fast and reproducible assessment of the RNFL and does not require a reference plane for the measurements. An initial study by Tjon-Fo Sang and Lemij found a high diagnostic accuracy for glaucoma detection with a sensitivity of $96 \%$ and a specificity of $93 \%{ }^{21}$ Subsequent studies, however, have found lower values of sensitivity and specificity. ${ }^{22}{ }^{23}$ Trible and coworkers ${ }^{23}$ found scanning laser polarimetry helpful in the differentiation of normal subjects from patients with glaucomatous damage. However, this test failed to detect a substantial number of subjects with severe damage, and the authors suggested caution before using the test as a screening method for glaucoma.

The evaluation of patients with band atrophy and temporal hemianopia represents an important model in the evaluation of the ability of the GDx to quantify nerve fibre layer loss. Its main advantage is the predictability of nerve fibre layer loss in 
such patients, particularly in the nasal and temporal regions of the optic nerve. Since the visual field defects in our patients were severe and long standing, and band atrophy was observed on fundus examination in all cases, a severe or complete loss of nerve fibres in the temporal and nasal portions of the optic disc may be inferred. Thus, it is clear that a severe loss of RNFL must have occurred in the nasal 70 degrees and in the temporal 50 degrees evaluated by the equipment. Our results, however, demonstrated that the GDx was not able to identify RNFL loss in the temporal region since there was no significant difference in RNFL thickness measurements between patients with band atrophy and controls. Moreover, the deviation from normal analysis did not identify abnormality in the temporal region of the patients with band atrophy.

The evaluation of the nasal region showed that although the mean RNFL thickness values were significantly different between the two groups, the deviation from normal analysis identified abnormality in only two of the 19 eyes with band atrophy (Fig 2). On the other hand, the significant loss of RNFL found in the superior and inferior portions of the disc seems to reflect the findings of Mildelberg and Yidegiligne ${ }^{16}$ who observed loss of fibres in these areas in an eye with band atrophy studied with automated quantitative histological analysis. These authors documented that, although the loss of nerve fibres occurs predominantly in the nasal and temporal segments, the superior and inferior areas of the optic disc are also involved. Our results are in accordance with those from many other investigators who consider the GDx sensitive enough to detect superior and inferior RNFL loss. ${ }^{261213}$

Previous studies have also questioned the ability of the GDx to identify nerve fibre layer loss in the temporal and nasal regions. Kogure and colleagues ${ }^{12}$ reported that the RNFL thickness measured on the superior and inferior regions were correlated with visual field change, but not on the temporal side of the optic disc. In another report, the same authors showed that there was no difference between the RNFL thickness of the temporal region in severe glaucoma eyes and that of normal, age matched controls. ${ }^{24}$ In a histological study, Morgan and coworkers ${ }^{25}$ compared the thickness of the RNFL around the optic disc in a primate eye with retardation measurements obtained in vivo using scanning laser polarimetry. A regional comparison showed that the best correlations occurred inferior and superior to the optic disc, with less positive correlation seen temporally. No significant correlation was found at the nasal aspect of the disc.

The diminished validity of measurements by the GDx on the nasal and temporal regions may result from incorrect compensation of anterior segment birefringence. While the RNFL exhibits substantial birefringence, it is not the only birefringent structure of the eye. The cornea and, to a lesser extent, the lens also exhibit birefringence. ${ }^{26}$ As all birefringent structures cause a change in the polarisation of the illuminating laser beam, the accuracy of RNFL thickness assessment with scanning laser polarimetry depends on the ability to extract the RNFL retardation from the total retardation. The GDx incorporates a cornea polarisation compensator designed to cancel the polarisation effects of the cornea. ${ }^{27}$ This compensator has a fixed optic axis ( $15^{\circ}$ nasally downward) and a fixed magnitude of retardation (60 $\mathrm{nm}$, single pass retardance), based on values found in the mode of the population. Therefore, the corneal component of retardation is eliminated only if the axis and magnitude of retardation of the cornea in the eye being imaged are identical to the mode of the population. However, several studies have shown that there is a wide variability of individual eyes both in the axis and in the magnitude of retardation. ${ }^{28}{ }^{29}$ Inadequate compensation of anterior segment birefringence may cause the total retardation measured by the GDx to reflect not only the RNFL retardation, but also the retardation of the combined cornea and corneal compensator. The magnitude of the minimum retardation around the optic disc (in the temporal and nasal parts of the peripapillary retina) is related to the magnitude of retardation arising from anterior birefringent structures. ${ }^{30}$ In fact, Zangwill and colleagues ${ }^{31}$ showed that individual correction of the corneal polarisation axis and magnitude improves the discriminating ability of the GDx. Using a variable corneal compensator, several parameters including those related to nasal and temporal thickness measurements showed improved ability for discriminating between normal and glaucomatous eyes. $^{31}$

The erroneous compensation of anterior segment birefringence produces a wider range of retardation measurements for the normal population, which may complicate the identification of abnormalities. ${ }^{30}$ This would explain, at least in part, the poor sensitivity of the deviation from normal analysis in detecting RNFL loss in the nasal and temporal regions of patients with band atrophy. However, we have no reasons to believe that there was any difference in anterior segment birefringence between the patients with band atrophy and the normal controls, other than by chance. Therefore, it is hardly likely for the anterior birefringence to have influenced the comparison between mean RNFL thicknesses in the two groups. As we were not able to detect a significant difference in the temporal RNFL thickness between the two groups, the poor sensitivity of the GDx in identifying loss of nerve fibres in this sector is probably associated with other factors.

Lee and Mok, ${ }^{6}$ studying patients with glaucoma using the GDx, found RNFL loss in the superior and inferior portions of the optic disc that was significantly different from the findings of normal patients. These authors, however, did not observe RNFL loss in the temporal and nasal portions of the optic disc and concluded that these areas were not affected by the disease. Our study is important in this regard because it shows that the findings obtained in the nasal and temporal portions of the disc should be interpreted with caution, since the GDx seems to lack the sensitivity required to detect abnormalities in those regions. This conclusion is especially important when studying optic neuropathies with a predilection for the nasal and temporal portions of the optic disc, particularly the temporal side which may be affected by a number of optic neuropathies including band atrophy, heredodegenerative diseases, toxic, carential, compressive and even inflammatory optic neuropathies. Failure to recognise the equipment deficiency in measuring RNFL in those locations can lead to erroneous diagnosis, a fact that should be taken into account by all physicians employing this semiological method.

\section{Authors' affiliations}

M L R Monteiro, F A Medeiros, M R Ostroscki, Department of Ophthalmology, University of São Paulo Medical School, São Paulo, Brazil

Correspondence to: Mário L R Monteiro, Av Angélica 1757 conj 61, 01227-200 São Paulo, SP, Brazil; mlrmonteiro@terra.com.br

Accepted for publication 18 July 2002

\section{REFERENCES}

1 Weinreb RN, Dreher AW, Coleman A, et al. Histopathologic validation of Fourier-elipsometry measurements of retinal nerve fiber layer thickness. Arch Ophthalmol 1990;108:557-60.

2 Weinreb RN, Shakiba S, Zangwill L Scanning laser polarimetry to measure the nerve fiber layer of normal and glaucomatous eyes. Am J Ophthalmol 1995;119:627-36.

3 Zangwill L, Berry CA, Garden VS, et al. Reproducibility of retardation measurements with the nerve fiber analyzer II. J Glaucoma 1997;6:384-9.

4 Hoh ST, Ishikawa H, Greenfield DS, et al. Peripapillary nerve fiber layer thickness measurement reproducibility using scanning laser polarimetry. $J$ Glaucoma 1998:7:12-15.

5 Colen TP, Tion-Fo-sang M, Mulder PG, et al. Reproducibility of measurements with the nerve fiber analyzer (NfA/GDx). J Glaucoma 2000;9:369-70.

6 Lee V WH, Mok KH. Retinal nerve fiber layer measurement by nerve fiber analyzer in normal subjects and patients with glaucoma. Ophthalmology 1999;106:1006-8. 
7 Lauande-Pimentel $\mathbf{R}$, Carvalho RA, Oliveira $\mathrm{HC}$, et al. Discrimination between normal and glaucomatous eyes with visual field and scanning laser polarimetry measurements. Br J Ophthalmol 2001;85:586-91.

8 Steel DH Waldock A. Measurement of the retinal nerve fibre layer with scanning laser polarimetry in patients with previous demyelinating optic neuritis. J Neurol Neurosurg Psychiatry 1998;64:505-9.

9 Medeiros FA, Susanna R Jr. Retinal nerve fiber layer loss after traumatic optic neuropathy detected by scanning laser polarimetry. Arch Ophthalmol 2001:119:920-1.

10 Colen TP, van Everdingen JAM, Lemij HG. Axonal loss in a patient with anterior ischemic optic neuropathy as measured with scanning laser polarimetry. Am J Ophthalmol 2000;130:847-50.

11 Meir FM, Bernasconi P, Stürmer J, et al. Axonal loss from acute optic neuropathy documented by scanning laser polarimetry. Br J Ophthalamol 2002;86:285-7

12 Weinreb RN, Shakiba S, Sample PA, et al. Association between quantitative nerve fiber layer measurement and visual field loss in glaucoma. Am J Ophthalmol 1995; 120:732-8.

13 Kogure S, lijima H, Tsukahara S. A new parameter for assessing the thickness of the retinal nerve fiber layer for glaucoma diagnosis. Eur $J$ Ophthalmol 1999;9:93-8.

14 Kogure S, Chiba T, Kinoshita T, et al. Effect of artifacts on scanning laser polarimetry of retinal nerve fibre layer thickness measurement. $\mathrm{Br} J$ Ophthalmol 2000;86:1013-1017.

15 Unsöld R, Hoyt WF. Band atrophy of the optic nerve: the histology of temporal hemianopia. Arch Ophthalmol 1980;98:1637-8.

16 Mikelberg FS, Yidegiligne HM. Axonal loss in band atrophy of the optic nerve in craniopharyngioma: a quantitative analysis. Can J Ophthalmol 1993;28:69-71.

17 Hoyt WF, Newman NM. The earliest observable defect in glaucoma? Lancet 1972; 1:692-3

18 Weinreb RN, Dreer AW, Bille JF. Quantitative assessment of the optic nerve head with the laser tomographic scanner. Int Ophthalmol $1989 ; 13: 25-9$
19 Zeimer RC, Mori MT, Khoobehi B. Feasibility test of a new method to measure retinal thickness noninvasively. Invest Ophthalmol 1989;30:2099-105

20 Schuman JS, Hee MR, Puliafito CA. Quantification of nerve fiber layer thickness in normal and glaucomatous eyes using optical coherence tomography. A pilot study. Arch Ophthalmol 1995;113:586-96.

21 Tjon-Fo, Sang M, Lemij HG. The sensitivity and specificity of nerve fiber layer measurements in glaucoma as determined with scanning laser polarimetry. Am J Ophthalmol 1997;1 23:62-9.

22 Weinreb RN, Zangwill L, Berry CC, et al. Detection of glaucoma with scanning laser polarimetry. Arch Ophthalmol 1998;116:1583-9.

23 Trible JR, Schultz RO, Robinson JC, et al. Accuracy of scanning laser polarimetry in the diagnosis of glaucoma. Arch Ophthalmol 1999;117:1298-304.

24 Kogure S. Assessment of the macula with scanning laser polarimetry. Jpn J Clin Ophthalmol 1999;53:273-6.

25 Morgan JE, Waldock A, Jeffery G, et al. Retinal nerve fibre layer polarimetry: histological and clinical comparison. $\mathrm{Br} J$ Ophthalmol 1998:82:684-90.

26 Van Blokland GJ, Verhelst SC. Corneal polarization in the living human eye explained with a biaxial model. J Opt Soc Am A 1987;4:82-90.

27 Dreher AW, Reiter K. Scanning laser polarimetry of the retinal nerve fiber layer. SPIE 1992;1746:34-41.

28 Greenfield DS, Knighton RW, Huang XR. Effect of corneal polarization axis on assessment of retinal nerve fiber layer thickness by scanning laser polarimetry. Am J Ophthalmol 2000;129:715-22.

29 Knighton RW, Huang XR. Linear birefringence of the central human cornea. Invest Ophthalmol Vis Sci 2002;43:82-6.

30 Garway-Heath DF, Greaney M, Caprioli J. Correction for the erroneous compensation of anterior segment birefringence with the scanning laser polarimeter for glaucoma diagnosis. Invest Ophthalmol Vis Sci 2002;43: 1465-74.

31 Zangwill LM, Bowd C, Greenfield DS, et al. Correction of corneal polarization axis and magnitude improves discriminating ability of GDx Nerve Fiber Analyzer. Presented at ARVO, Fort Lauderdale, 2002. Abstract no 930.

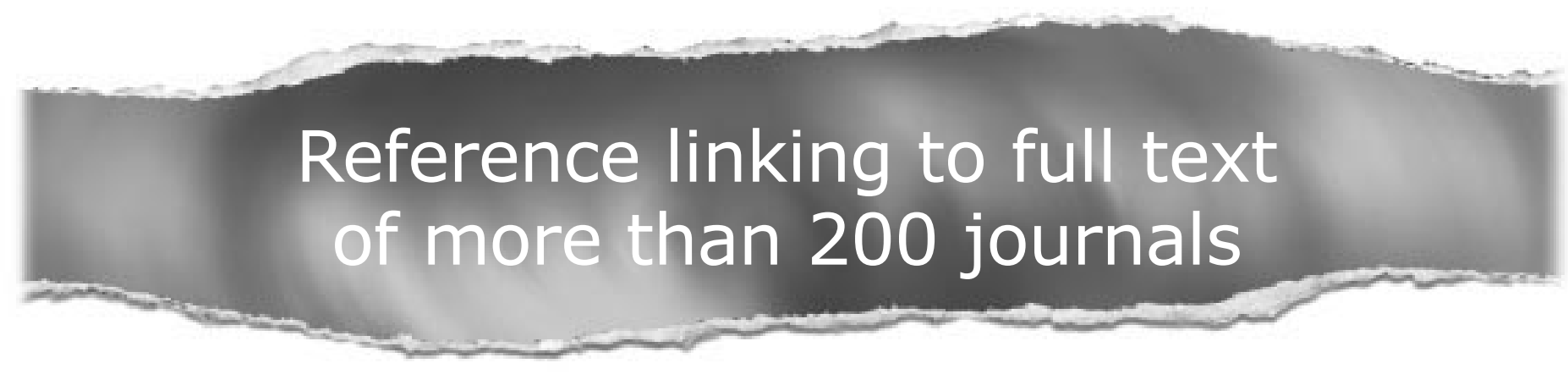

Toll free links

You can access the FULL TEXT of articles cited in the British Journal of Ophthalmology online if the citation is to one of the more than 200 journals hosted by HighWire (http://highwire.stanford.edu) without a subscription to that journal. There are also direct links from references to the Medline abstract for other titles.

www.bjophthalmol.com 